\title{
Tree Sap Induced Corneal and Lens Crystals
}

\author{
Sana Nadeem
}

Pak J Ophthalmol 2019, Vol. 35, No. 4

See end of article for authors affiliations

Correspondence to:

Dr. Sana Nadeem

Assistant Professor

Email:

sana.nadeem@fui.edu.pk,
This is a peculiar case of localized, silvery, iridescent crystal deposition in the cornea and lens, discovered twenty seven years after injury with an overhanging tree branch with sap exposure. The corneal tear at the time of injury was self sealing and there was also associated lens trauma, both managed conservatively on an inpatient basis, and leading subsequently to a corneal scar and localized cortical cataract, respectively, both demonstrating fine, silver, interspersed crystals.

Key Words: Crystals, cornea, lens, trauma, cataract, corneal scar.
$\mathrm{P}$ lant trauma may exert its effects by mechanical forces, chemical injury or a hypersensitivity reaction. Crystalline keratopathy due to plant sap exposure was reported for the first time in 1973 by Ellis ${ }^{1}$, who described needle-like crystals in the corneal epithelium and stroma in a boy, who accidentally splashed his eyes with the sap of Dieffenbachia, an ornamental houseplant. These needle-like crystals or raphides ${ }^{2}$, are found within the leaves and stems of this plant, and are composed of calcium oxalate, which cause an explosive keratoconjunctivitis upon contact with eyes.

The other species3,4 of plants known to cause crystalline keratopathy are Arisaema, Colocasia, Pinellia, Phylodendron, and Alocasia, although raphides are also found in other philodendron plants of the Araceae family, and may cause similar findings. ${ }^{2}$ The more common cause of crystal deposition in the cornea is infectious crystalline keratopathy, first reported in 1983 by Gorovoy ${ }^{5}$ et al in a corneal transplant, attributed to stromal colonization of gram-positive cocci along a suture tract. Subsequently, there have been numerous such cases, classically described as branching, needle-like stromal crystals; associated most frequently with penetrating keratoplasty, but also with incisional surgeries like keratotomy, contact lens wear, chemical burns, and even topical anesthetic abuse.6,7 Culprits identified are most commonly bacteria; Streptococcus viridians most often, but S. pneumoniae, Haemophilus aphrophilus, Peptostreptococcus, Pseudomonas aeruginosa, and numerous others; but fungi like Candida and Alternaria species have been isolated as well ${ }^{6}$.

Schnyder corneal dystrophy, cystinosis, tyrosinemia, gout, Bietti crystalline dystrophy, multiple myeloma, monoclonal gammopathy, gold, and drugs, are other causes of corneal crystals ${ }^{8}$. Scant case reports of self-induced corneal crystals can also be found, with one man admitting to injecting blue eyeshadow $^{9}$ into his corneas. However, a crystalline traumatic cataract has never been described before in literature. We report a case of crystalline keratopathy with associated similar crystalline cataract as a result of tree sap injury, decades prior to discovery.

\section{CASE REPORT}

A 51-year-old hospital staff worker with no known comorbids presented in the outpatient department of Fauji Foundation Hospital, which is a tertiary care teaching hospital, affiliated with the Foundation University Medical College; with complaints of 
grittiness and redness of the left eye for a few days. On examination, his visual acuity was $6 / 6$ in the right eye and 6/12 in the left; his symptoms were attributed to an inflamed pterygium of the left eye. However, it was discovered on routine slit lamp examination, that his left cornea had a sickle shaped distribution of fine, iridescent, silvery crystals in a full thickness, paracentral corneal scar extending from 5:30 to 8:30 o'clock (Figure $1 \mathrm{~A}-\mathrm{E})$.
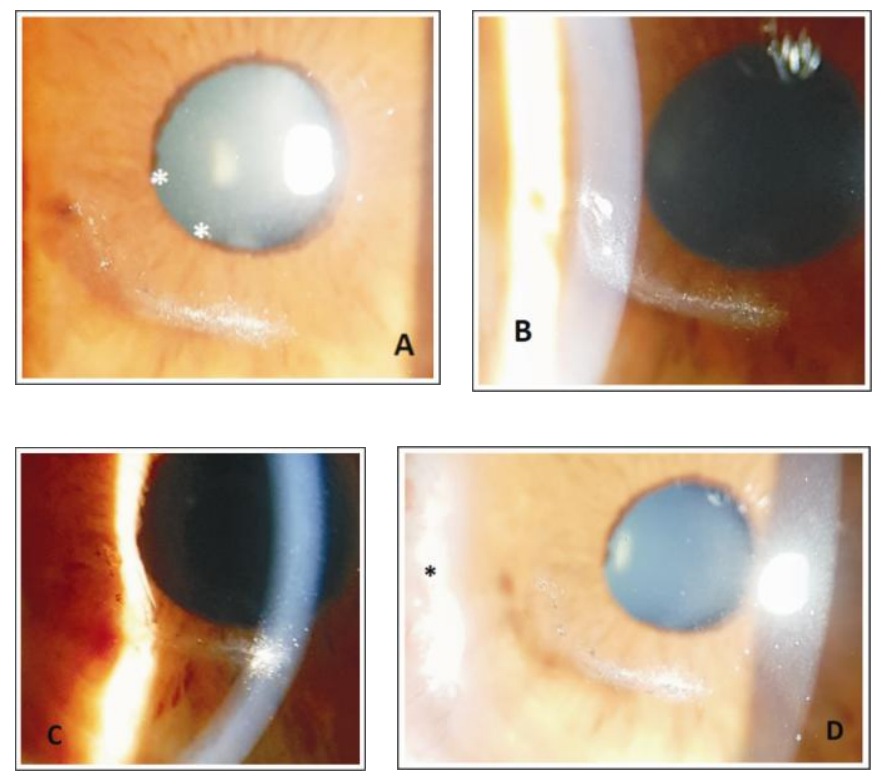

Fig. 1: A. Corneal crystals in a sickle shaped scar and sphincter damage (white asterisks) B. Larger deposits on lateral illumination C. Full thickness crystal deposition D. Inflamed pterygium (black asterisk) and brownish deposits intermixed with crystals.

Brownish deposits were also seen at some places intermixed with the crystals. This finding was correlated by the patient, to a tree branch injury which occurred in 1991, twenty seven years back, when he got hit accidentally, by an overhanging tree branch while travelling on the roof of a large vehicle at night. He did not see the tree in the dark and could not identify the type of tree; however, he did recall some substance being instilled in his eye leading to severe inflammatory symptoms. He was rushed to and admitted at that time in a local hospital and was managed conservatively with topical and systemic antibiotics or steroids, presumably, because he was unaware of the nature of the medicines and could not recall their names. He denied self-medication or instillation of any chemical into his eye. On further examination, he had a quiet anterior chamber, but iris sphincter damage was visualized from $7 \mathrm{o}$ clock to 8:45 o' clock, with loss of the pupillary ruff, and a hint of a localized cataract. Upon dilatation of pupil, similar iridescent crystals were seen dispersed inside the localized oval, traumatic cortical cataract at $5 \mathrm{o}^{\prime}$ clock position (Figure 2).



Fig. 2: Iridescent crystalline traumatic cortical cataract.

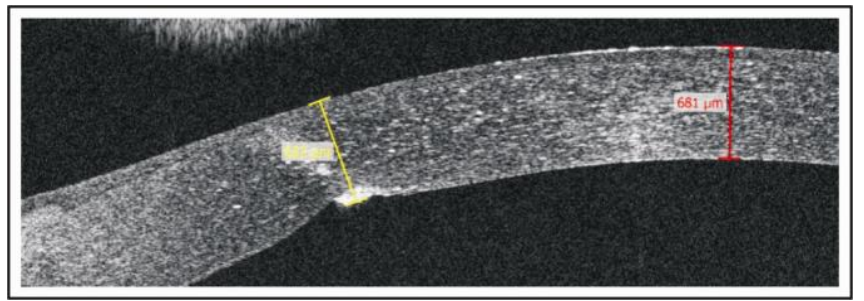

Fig. 3: Anterior segment OCT (AS-OCT) of the left cornea, showing the full thickness scar measuring $652 \mu \mathrm{m}$, with refractile crystals dispersed in the stroma. The central corneal thickness is $681 \mu \mathrm{m}$.

The rest of the lens was clear. Anterior segment OCT (AS-OCT) was done which showed full thickness corneal scar, with stromal dispersion of crystals (Figure 3), and the cortical lenticular opacity with crystals as well (Figure 4). 

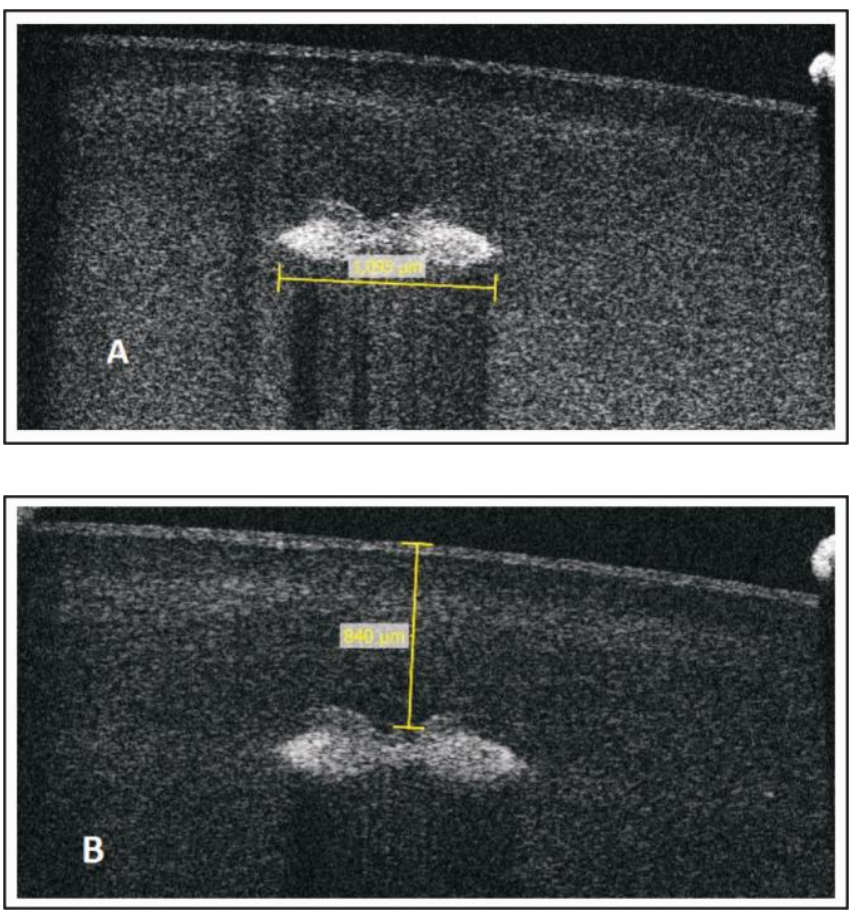

Fig. 4: A. AS-OCT of the lens showing the anterior cortical cataract $(1095 \mu \mathrm{m}$ in size), with interspersed crystals.

B. Cataract is located $840 \mu \mathrm{m}$ from the anterior lens capsule.

The fundus examination was normal. The right was normal except for a mild pterygium. Intraocular pressures were $11 \mathrm{~mm} \mathrm{Hg}$ OD and $12 \mathrm{~mm} \mathrm{Hg}$ OS. He was given topical tobramycin-dexamethasone drops thrice a day for two weeks for the inflamed pterygium.

Routine investigations performed were blood complete picture, random blood sugar, renal function tests, and Urine routine examination, which were all normal.

The crystals did not interfere with vision, nor seemed infectious, so were not sought to be treated, neither was the traumatic, crystalline cataract, which was not in the pupillary area. The crystals were stable until the last follow-up.

\section{DISCUSSION}

Trees and plants with milky sap are a common place. Such plants can be ornamental, or used in medicines or food. About 12 families, 20 genera and more than 5000 species of milky latex sap occur in the world. The toxicity from the sap is attributed to essential oils, alkaloids, amino acids, proteins, glycerides, plant acids, peptides, saponins, terpenes, furano-coumarins, and poly-acetylene compounds. Local or oral use can have profound toxic effects. Skin contact can lead to a blistering reaction, while contact with eyes can cause a severe keratoconjunctivitis, uveitis, corneal scarring and even permanent visual loss 9,10 .

Dieffenbachia plant typically has ejector pods containing raphides, which if lightly squeezed, result in an explosive ejection ${ }^{2}$ of these needle-like calcium oxalate crystals, which penetrate the corneal epithelium; allowing further chemical injury by oxalic acid and plant proteins. This gun like effect allows these crystals to penetrate deep into the cornea.

All the six species of the Araceae family ${ }^{1-4}$; Dieffenbachia, Arisaema and Colocasia, Alocasia, Pinellia, and Phylodendron; reported previously to cause corneal crystals, are small ornamental plants, but in this case, our patient was hit by a tall tree, whose nature is obviously unknown to us; and could, but possibly does not belong to this family; as these are small ornamental plants, and not at all tall. We would have liked to identify the tree, but the injury occurred decades ago, in the dark night, and the patient could not identify or recall the type of tree at all, and also because of the severe symptoms caused by it, he was rushed to a hospital. The offending plant caused a penetrating injury to the eye, resulting in a full thickness corneal tear and the sap penetrated both the cornea and the lens capsule as well, to cause crystallization in both the corneal stroma, and also within the small, cortical cataract.

Although, plants from the Araceae family are found in Pakistan ${ }^{10}$, it is difficult in this case to identify the offending plant.

Dieffenbachia typically causes fine, blue9, needle like crystals within the corneal stroma, which resolve with topical steroid and antibiotic therapy. Our patient had fine and silvery crystals, rather than needle-like, which are probably not of the same nature as calcium oxalate. Deposition of these crystals deep into the anterior lens cortex, also suggests an explosive mechanism of ejection of plant sap, similar to the Dieffenbachia plant. The crystals in our patient differ from other causes of crystalline keratopathy, especially infectious; where the crystals are also needle like. Association of the crystals to the corneal scar and cataract point to tree sap injury, rather than other metabolic causes or drugs, which lead to bilateral deposits, and have been ruled out on the basis of history and investigations. 
Plant sap exposure is an extremely interesting cause of corneal crystals, and in this case lenticular crystals as well; which is unknown to many ophthalmologists, and must be kept in mind while evaluating a patient who presents to us with corneal crystals. In addition, the ophthalmologists need to be aware of the constituents of these milky sap plants and their sequelae, in order to effectively treat such cases. The patients should be asked to identify the culprit plant and bring the offending leaf with them. Upon identification, it is important to report these plants in order to increase awareness and prevent injury.

The need arises to wear protective glasses and gloves while working in the garden, and to rinse the splashed areas immediately, in the event of accidental exposure. Such patients with eye exposure presenting to the hospital need to be irrigated with normal saline immediately, followed by meticulous steroid and antibiotic therapy to suppress keratoconjunctivitis, which in the majority of cases is self-limiting?.

In conclusion, crystals in the eye have abundant causes, the common ones have been discussed frequently. However, cataract inflicted by tree sap injury must also be kept in mind while evaluating these cases.

\section{Author's Affiliation}

\section{Dr. Sana Nadeem}

Assistant Professor, Department of Ophthalmology, Foundation University Medical College/Fauji Foundation Hospital, Rawalpindi

\section{REFERENCES}

1. Ellis W, Barfort P, Mastman GJ. Keratoconjunctivitis with corneal crystals caused by the diffenbachia plant. Am J Ophthalmol. 1973 Jul; 76 (1): 143-147.

2. Seet B, Chan WK, Ang CL. Crystalline keratopathy from Dieffenbachia plant sap. Br J Ophthalmol. 1995; 79: 98-99.

3. Tang EW, Law RW, Lai JS. Corneal injury by wild taro. Clin Exp Ophthalmol. 2006 Dec; 34 (9): 895-6.

4. Hsueh KF, Lin PU, Lee SM, Hsieh CF. Ocular injuries from plant sap of genera Euphorbia and Dieffenbachia. J Chin Med Assoc. 2004; 67 (2): 93-98.

5. Gorovoy MS, Stern GA, Hood CI, Allen C. Intrastromal noninflammatory colonization of a corneal graft. Arch Ophthalmol. 1983 Nov; 101 (11): 1749-52.

6. Yanoff M, Duker JS. Ophthalmology. Third Edition. Mosby: St. Louis, 2009: p 264.

7. Porter AJ, Lee GA, Jun AS. Infectious crystalline keratopathy. Surv Ophthalmol. 2018 Jul-Aug; 63 (4) 480-499.

8. Weiss JS, Khemichian AJ. Differential diagnosis of Schnyder crystalline dystrophy. Dev Ophthalmol. 2011; 48: 67-96.

9. Lembach RG, Ringel DM. Factitious bilateral crystalline keratopathy. Cornea, 1990 Jul; 9 (3): 246-8.

10. Ahmad S. A study of poisonous plants of Islamabad area, Pakistan. Pak J Sci Ind Res. Ser. B. 2012; 55 (3): 129 137.

\section{Author's Contribution}

\section{Dr. Sana Nadeem}

Data collection, manuscript design, literature review, final review. 